\title{
Chronological Improvement in Survival Following Rectal Cancer Surgery: A Large-Scale, Single-Center Study
}

\author{
Jong Lyul Lee • Chang Sik Yu • Chang Wook Kim • \\ Yong Sik Yoon · Seok-Byung Lim • \\ Jin Cheon Kim
}

Published online: 23 October 2013

(C) Société Internationale de Chirurgie 2013

The authors greatly appreciate the recent publication of our article titled Chronological Improvement in Survival Following Rectal Cancer Surgery: A Large-Scale, SingleCenter Study [1] in the July 31, 2013, issue online. However, one coauthor of our report has expressed concern that some readers may confuse the oncologic outcomes reported in this article with the results presented in previous articles from our institution.

The following articles from our institution report associations with oncologic outcomes for the patients with rectal cancer (main content/study period/number of patients in overlapping reports): (a) adjuvant chemoradiotherapy vs lateral pelvic lymph node dissection/ 2004-2006/146 patients [2]; and (b) abdominoperineal resection versus low anterior resection/1995-2005/573 patients [3]. Although a portion (a maximum of $26 \%$ ) of these study cohorts overlapped, each study design was different, and the inclusion criteria were different. A small difference in oncologic outcomes may be the result of differences in the study inclusion criteria. Unlike the first of these previous studies [2], one third of the patients in our study did not receive adjuvant radiotherapy. Also, our investigation was not a matched case-control study, which distinguishes it from the second report [3]. In contrast, we evaluated all patients who underwent radical surgery for stage II or III rectal cancer during the study period.

We wish to clarify the basis for the differences in oncologic outcomes between our report and other articles from our institution and to remove readers' possible confusion about the differences in oncologic outcomes.

All authors are aware of and agree to this notification.

\section{References}

1. Lee JL, Yu CS, Kim CW, et al (2013) Chronological improvement in survival following rectal cancer surgery: a large-scale, singlecenter study. World J Surg 37:2693-2699

2. Kim JC, Takahashi K, Yu CS et al (2007) Comparative outcome between chemoradiotherapy and lateral pelvic lymph node dissection following total mesorectal excision in rectal cancer. Ann Surg 246:754-762

3. Kim JC, Yu CS, Lim SB et al (2013) Abdominoperineal resection and low anterior resection: comparison of long-term oncologic outcomes in matched patients with lower rectal cancer. Int $\mathbf{J}$ Colorectal Dis 28:493-501
J. L. Lee · C. S. Yu - C. W. Kim · Y. S. Yoon - S.-B. Lim ·

J. C. Kim

Department of Surgery, University of Ulsan College of

Medicine, Seoul, Republic of Korea

C. S. Yu $(\bowtie)$

Asan Medical Center, 86, Asanbyeongwon-gil, Songpa-gu,

Seoul 138-736, Republic of Korea

e-mail: csyu@amc.seoul.kr 